University of Nebraska - Lincoln

DigitalCommons@University of Nebraska - Lincoln

Faculty Publications: Materials Research

Science and Engineering Center

Materials Research Science and Engineering

Center

2006

\title{
Understanding and Control of Lateral Contraction in Stamping Lithography
}

Zheng Li

Peking University, Beijing, China

Li Tan

University of Nebraska-Lincoln, Itan4@unl.edu

Gang-Yu Liu

University of California, Davis

Follow this and additional works at: https://digitalcommons.unl.edu/mrsecfacpubs

Part of the Materials Science and Engineering Commons

$\mathrm{Li}$, Zheng; Tan, Li; and Liu, Gang-Yu, "Understanding and Control of Lateral Contraction in Stamping Lithography" (2006). Faculty Publications: Materials Research Science and Engineering Center. 75. https://digitalcommons.unl.edu/mrsecfacpubs/75

This Article is brought to you for free and open access by the Materials Research Science and Engineering Center at DigitalCommons@University of Nebraska - Lincoln. It has been accepted for inclusion in Faculty Publications: Materials Research Science and Engineering Center by an authorized administrator of DigitalCommons@University of Nebraska - Lincoln. 


\title{
Understanding and Control of Lateral Contraction in Stamping Lithography
}

Zheng $\mathrm{Li}^{1}$, Li Tan ${ }^{2}$, and Gang-yu Liu ${ }^{3}$

${ }^{1}$ Department of Mechanics and Engineering Science, Peking University, Beijing, China, People's Republic of

${ }^{2}$ Department of Engineering Mechanics and Nebraska Center for Materials and Nanoscience, University of Nebraska, Lincoln, NE, 68516

${ }^{3}$ Department of Chemistry, University of California, Davis, CA, 95616

\begin{abstract}
Thin film contraction under external mechanical stress can be used to miniaturize size and increase density of patterned features on top. Nonlinear Finite Element Analysis is used to provide guidance on this contraction process. It was found that the substrate contraction causes stress accumulation along interfaces between protruded features and substrate. These stress accumulation complexes the control of profile changes on patterned features and suggest a design of patterned features arranged beyond a critical distance to avoid cross-interference.
\end{abstract}

\section{INTRODUCTION}

Stamping lithography, including soft lithography [1] and imprint lithography [2], has made deep impacts on nanomanufacturing due to its nanometer scale resolution, easiness in operation and compatibility to a variety of materials. In this process, a master stamp is used to transfer topographic features into thin films, such that, either negative or positive replicas are obtained. Many efforts have been invested to afford manufacture flexibility to stamped features, in which small scale structures with size or shape different from those on the master stamp were demonstrated $[3,4]$. Pathways contributing to such flexibility mainly include a fine tuning of master stamp deformation in vertical direction, such as a multi-layered master stamp design [5] or a careful control on pressuring the soft stamp [6,7]. Feature size miniaturization, on the other hand, was performed through a designated dewetting or diffusion process on the stamped material $[8,9]$. Further attenuation of the replica structures, however, presents great challenges, such as complication in tuning feature density, lack of means for reducing line edge roughness, as well as a much concerned resolution in competing with beam or scanning probe lithography. Recently (2006) G. Y. Liu and others presented the concept of feature miniaturization through lateral contraction of the substrate [10], which shed light on addressing part of above challenges. Lateral contraction process, such as uni- or bi-axial contraction, was used to aggressively reduce feature size and increase feature density at the same time. For instance, sub-40 nm protein gratings are revealed after contracting original sub-micrometer features, and correspondingly pattern density has been increased 10 fold.

This added process in stamping suggests great flexibility in tuning both feature size and density, thus, suggesting less demand on precision manufacturing of the master stamp from other high-end lithography platforms. Since this lateral process involves a nonlinear and large-scale 
deformation in elastic membrane, difficulties to seek an analytical guidance are anticipated with conventional theoretical tools. To make this miniaturization process controllable with thorough understanding, preliminary work on modeling and simulation are provided in this paper. In particular, we discuss the change in pattern profile and density on elastic membrane and hope the investigation provide guidance on relating lateral deformation to patterned features, and more importantly, to better regulate pattern integrities.

\section{EXPERIMENTAL AND MODELING DETAILS}

Elastic material used is standard poly(dimethylsiloxane) (PDMS, Sylgard 184®) material from commercial source (Krayden Inc., Denver, CO). The PDMS membrane was formed by first spin-coating the pre-elastomer liquid (base:curing reagent $=10: 1$ ) on a smooth polycarbonate substrate at $500 \mathrm{rpm}$ for $30 \mathrm{~s}$; and then baked at $65^{\circ} \mathrm{C}$ for $2 \mathrm{hrs}$. The cured membrane was then peeled off from the substrate, carved to a long strip shape $(2 \mathrm{~cm} \times 0.5 \mathrm{~cm}$, thickness of $0.1 \mathrm{~mm}$ ) and elongated at $20 \mathrm{~mm} / \mathrm{s}$ to generate the stress-strain curve.

Nonlinear Finite Element Analysis (FEA) is used to simulate the lateral contraction, in which plane stress state or 3-D stress state in thin membrane is computed by ANSYS. The membrane structure is firstly divided by PLANE182 (2-D 4-node) or SOLID185 element (3-D 8-node) to represent the 2-D or 3-D stress state respectively. Since the miniaturization process involves nonlinear and large deformation, both elements are selected to be hyperelastic, with capabilities of large deflection and strain. Simulation is then carried out following the Newton-Raphson approach, where the load is subdivided into 10 load increments and each load step is subdivided into 100 substeps. In addition, convergence criteria based on force and displacement are utilized, and the line search option in ANSYS is activated to converge the numerical calculation.

\section{RESULTS AND DISCUSSION}

Figure 1 illustrates the added contraction process to conventional stamping lithography, where substrate contraction induces feature size reduction. Experimentally, patterned features are firstly created over an elongated membrane [10]. Then, these features are contracted after tensile stress on the membrane is released.

$\mathbf{a}$

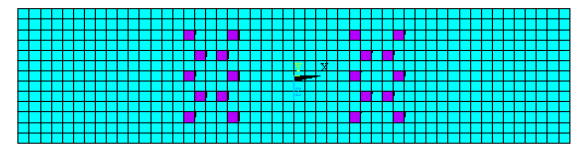

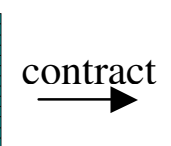

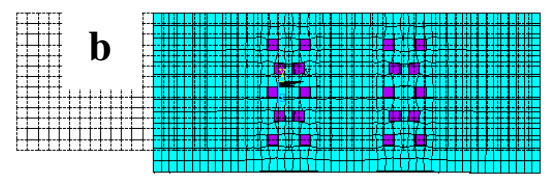

Figure 1. Schematic illustration of the contraction-induced pattern attenuation (top view).

\section{Hyperelastic model for elastic membrane}

Poly(dimethylsiloxane) (PDMS) is selected as the elastic substrate for the lateral deformation. Parameters of the constitutive model for such material are informed with curve 
fitting the stress-strain data. Since the elastic material is not compressible, a hyperelastic constitutive model (Ogden type) based on the existence of elastic strain energy potential is used to describe the associated deformation. The Ogden model [11] is given by

$$
W=\sum_{i=1}^{N} \frac{\mu_{i}}{\alpha_{i}}\left(\lambda_{1}^{\alpha_{i}}+\lambda_{2}^{\alpha_{i}}+\lambda_{3}^{\alpha_{i}}-3\right)
$$

where $\mu_{i}$ and $\alpha_{i}$ are material constants, $\lambda_{i}$ is the principal stretching ratio. Figure 2 shows a typical tension curve when a uni-axial stress is applied over a thin sheet of PDMS membrane. First-order parameters of $\mu_{1}=0.30 \mathrm{MPa}$ and $\alpha_{1}=4.92$ in Eq. 1 can thus be informed by curve fitting. These two parameters are then used to describe the hyperelastic deformation in the following sections.

\section{Modeling membrane and patterned structure contraction}

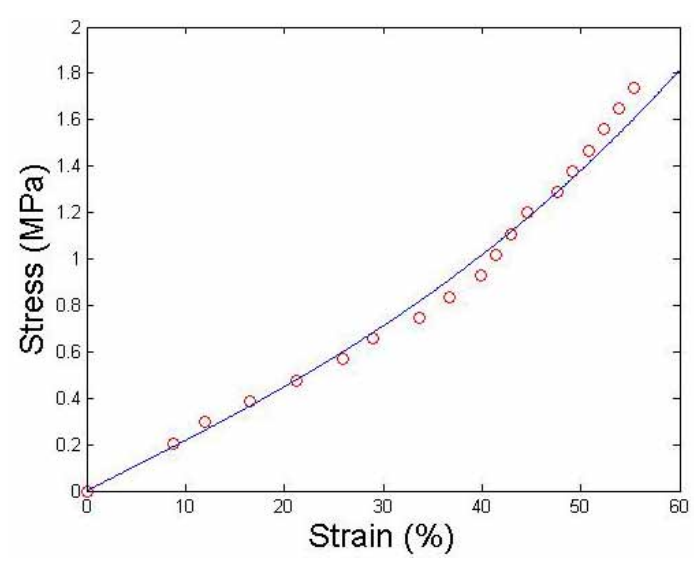

Figure 2. Uniaxial stretching of an elastic membrane (circle) and curve fitting (solid line) with Ogden model.

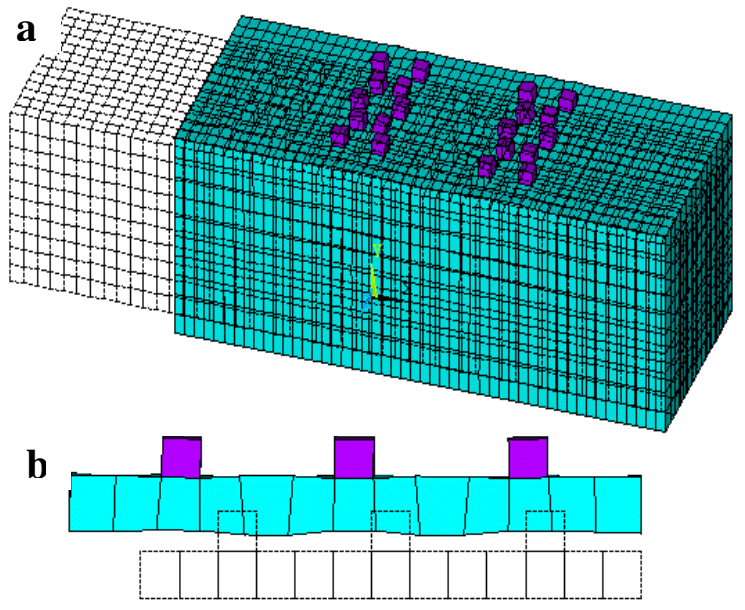

Figure 3. Rigid pattern contraction during lateral contraction of substrate.

Since the contraction of the elastic membrane adds a shearing process to patterned features, internal stress will be accumulated throughout the protruded patterns and consequently, profiles of these protrusions might vary depending on resistance of these materials to shearing. To analyze the deformation along these interfaces and its effects to pattern integrities, in the following section, either rigid (thermoplastic) or soft (elastic) polymer materials are selected to represent typical materials used in this process.

Figure 3 shows change of rigid structures after contraction of the soft membrane, where polystyrene (PS) particles with $360 \mathrm{~nm}$ diameter (SOLID185 element, 11502 nodes and 6523 elements) are dispersed over the PDMS membrane. The particles are assumed to have a Young's modulus of 3.2 GPa and Poisson's ratio of 0.33 [12]. Since these particles are rigid with high resistance to the mild shearing process, pattern profiles remain intact but surely add boundary to interrupt the smooth contraction of the elastic membrane if good adhesion between PS and PDMS is assumed. The interruption along PS/PDMS interfaces are shown on the side view in Figure 3(b). Clearly, PS particles on the membrane restrained the lateral deformation of elastic membrane, and such rigid restraint exhibits a surface fluctuation on membrane with a wavelength around $1.5 \mu \mathrm{m}$. 
Soft PDMS structure over elastic membrane, featured with grating lines of $360 \mathrm{~nm}$ in width and spacing of $720 \mathrm{~nm}$, is divided into 531 nodes and 464 elements (PLANE182) and illustrated in Figure 4. Generally, when the tensile force on membrane is released, soft grating lines are contracted and this contraction leads to lateral size reduction in patterned features not observed
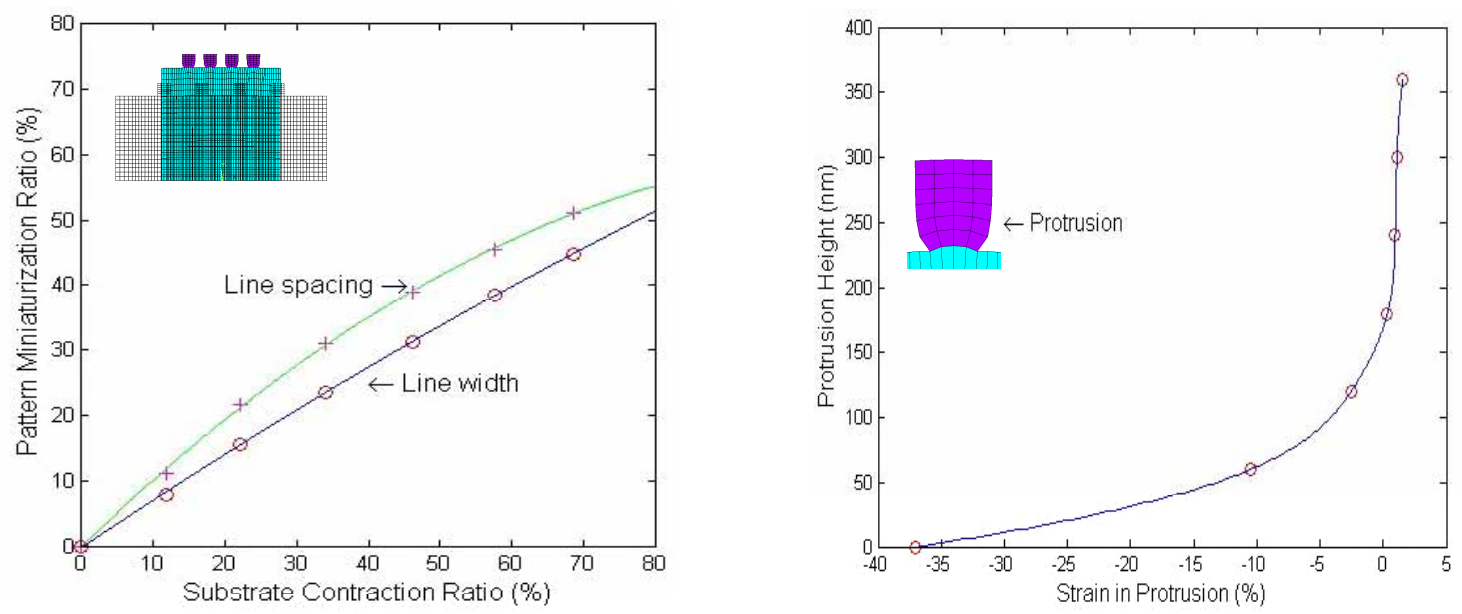

Figure 4. (a) Pattern miniaturization with the contraction of substrate and (b) contraction induced pattern profile change.
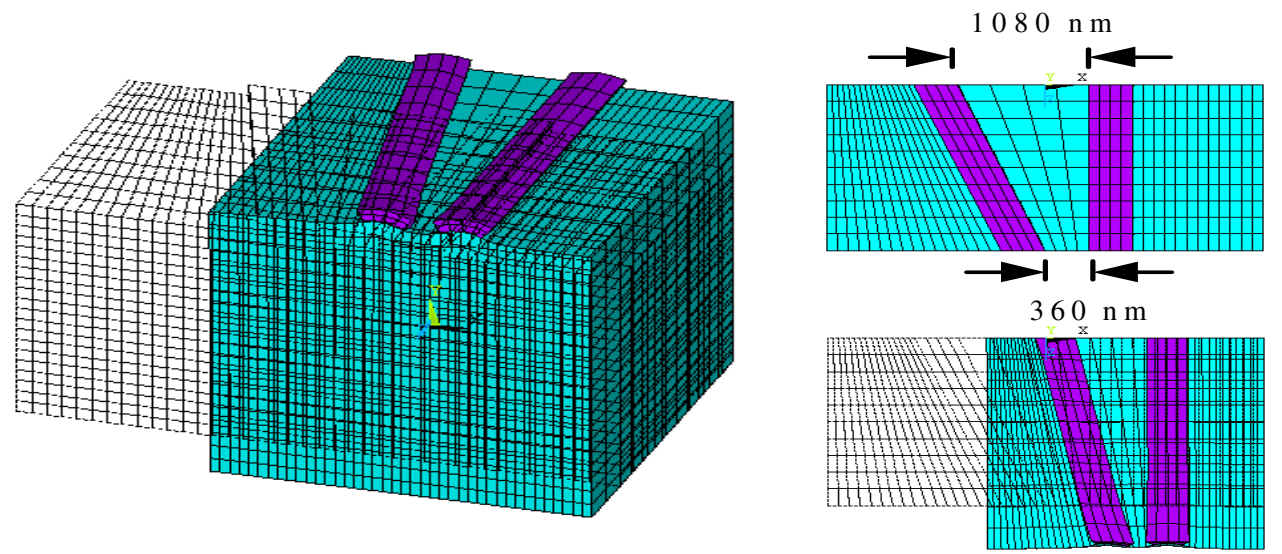

Figure 5. Pattern profile change during contraction for soft features on elastic membrane.

for rigid PS features (Figure 4(a)). It is also evident that the line spacing shrinks more significant than the soft patterned features. Since dimension reduction on x-direction of the substrate leads to a thickness increase of the substrate in z-direction to conserve volume, the corresponding pattern profile change on z-direction is expected and presented in Figure 4(b). Simulation indicates that the contraction process causes stress accumulation along interfaces between protrusion and substrate. In addition, the strain in protrusions varies with the height of the protrusion. This can be rationalized because the shearing force originates from the interface and thus, has less effect to structures farther away from the interface. Therefore, features closer to the membrane substrate are contracted much easier and a "neck" at the bottom of the protrusion is anticipated. The extent of such "necking" to pattern profile, however, awaits further experimental verification. 
Another significant merit in the contraction process is the capability to increase pattern density. Figure 3 shows the density change for rigid structures. Clearly, spacing between particle features is reduced while substrate contracts and no size reduction on protruded features is expected, thus, spacing contraction becomes the dominant factor for pattern density increase. When soft materials are used for structuring over the elongated elastic membrane, both feature size and spacing reduction will lead to pattern density increase during substrate contraction. Figure 5 shows such an example, where two non-equidistant lines (SOLID185 element, 11319 nodes and 8160 elements) with $360 \mathrm{~nm}$ in width and $100 \mathrm{~nm}$ in height are spaced between 360 and $1080 \mathrm{~nm}$. Line features on this substrate are compressed when the substrate contracts $(\sim 46 \%)$, reduced distance and angle between the two line features are revealed.

It is worthwhile to note that the change on pattern profile is affected by the spacing in between. Figure 6(a) indicates that when lines are spaced more than $600 \mathrm{~nm}$, uniform pattern profile reduction exists as displayed by the constant strain curve. In contrast, when the spacing falls in the range of $360-600 \mathrm{~nm}$, strain inside the features start to fluctuate and thus, crossinterference between features is evident and this lead to variation on pattern profile change. Certainly, this dependence on spacing is governed by the bottom surface fluctuation which is a result of volume restraint from the patterned feature. This critical line spacing for crossinterference is probably one such fluctuation wave, which has a wavelength about $600 \mathrm{~nm}$ as shown from the data in Figure 6(a). The corresponding pattern profile change is shown in Figure 6(b), in which profile of the front pattern (with $360 \mathrm{~nm}$ spacing) shows stronger deviation from the ideal square cross-section as comparing to the rear pattern (with $1080 \mathrm{~nm}$ spacing).

Interestingly, the occurrence of such fluctuation and its cross-interference is sensitive to the boundary materials on top. For instance, a significant surface fluctuation $(\sim 1.5 \mu \mathrm{m}$ in horizontal direction) is observed for PS/PDMS structures with the feature dimension of $360 \mathrm{~nm}$.
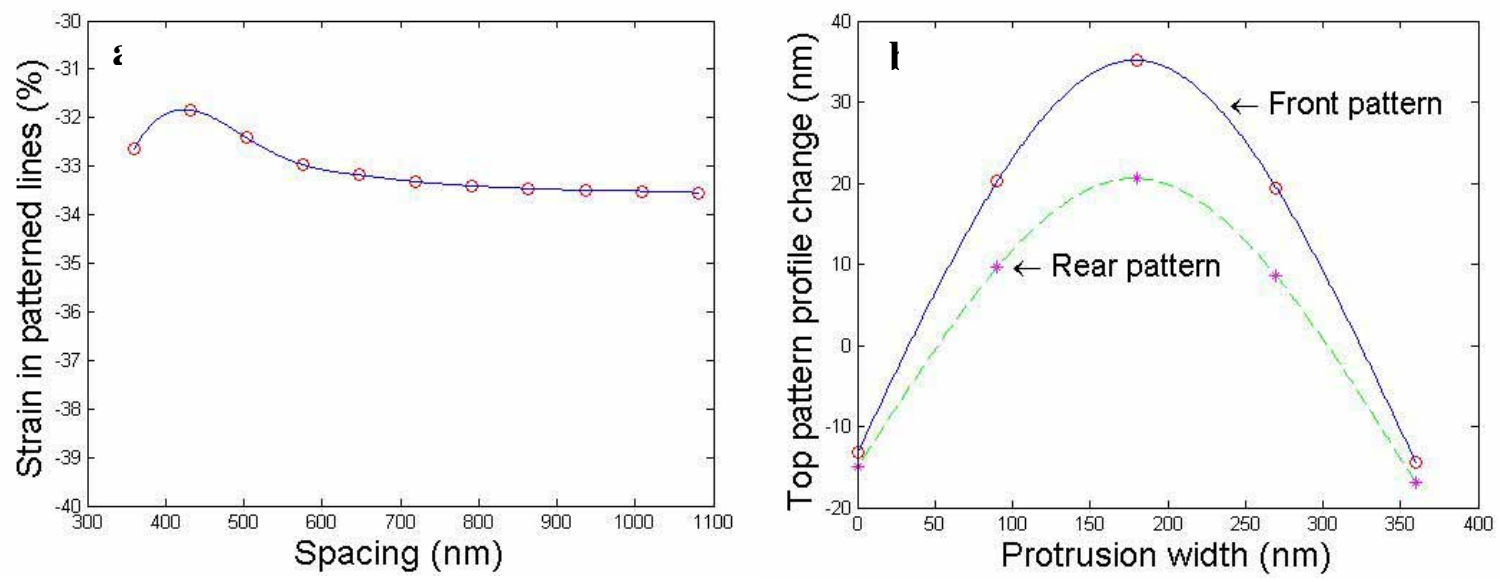

Figure 6. (a) Strain dependence on feature spacing in miniaturized patterns and (b) crossinterference effect to pattern profile change after lateral contraction.

\section{CONCLUSIONS}

This study shows that lateral contraction in stamping lithography can be modeled and simulated using nonlinear Finite Element Analysis. It was found that the substrate contraction causes stress accumulation along interfaces between protruded features and substrate. These 
stress accumulation complexes the profile changes on soft features and suggest a design of patterns arranged beyond a critical distance to avoid cross-interference. This analysis provides preliminary guidance on relating lateral deformation to control the miniaturization process.

\section{ACKNOWLEDGMENTS}

The authors would like to thank Prof. Mehrdad Negahban for critical discussion. The project described was made possible by Nebraska Tobacco Settlement Biomedical Research Development Fund, Charles J. Millard Trust Fund and Nebraska EPSCoR first award. One of the authors, Dr. Zheng Li, was partially supported by the exchange program between Peking University and the University of Nebraska-Lincoln.

\section{REFERENCES}

1. Y. N. Xia and G. M. Whitesides, Annu. Rev. Mater. Sci. 28, 153 (1998).

2. S. Y. Chou, P. R. Krauss, and P. J. Renstrom, J. Vac. Sci. Technol. B 14, 4129 (1996).

3. Y. Xia, E. Kim, X.-M. Zhao, J.A. Rogers, M. Prentiss and G.M. Whitesides, Science 273, 347 (1996).

4. J.A. Rogers, O.J.A. Schueller, C. Marzolin and G.M. Whitesides, Appl. Optics 36, 1-4 (1997).

5. J. Tien, C. M. Nelson, and C. S. Chen, Proc. Natl. Acad. Sci. USA 99, 1758 (2002)

6. L. Tan, Y. P. Kong, L. R. Bao, X. D. Huang, L. J. Guo, S. W. Pang, and A. F. Yee, J. Vac. Sci. Technol. B 21, 2742 (2003)

7. A. Bietsch and B. Michel, J. Appl. Phys. 88, 4310 (2000)

8. L. R. Bao, L. Tan, X. D. Huang, Y. P. Kong, L. J. Guo, S. W. Pang, and A. F. Yee, J. Vac. Sci. Technol. B 21, 2749 (2003)

9. P. Klajn, M. Fialkowski, I. T. Bensemann, A. Bitner, C. J. Campbell, K. Bishop, S. Smoukov, and B. A. Grzybowski, Nature Mater. 3, 729 (2004)

10. Z. Ouyang, L. Tan, M. Liu, O. Judge, X. Zhang, H. Li, J. Hu, T. E. Patten, and G. Y. Liu, Small, 2, 884 (2006)

11. R. W. Ogden, "Non-linear Elastic Deformations", Ellis Horwood Limited, pp. 204-222, 1984

12. L. E. Nielsen and R. F. Landel, "Mechanical Properties of Polymers and Composites", $2^{\text {nd }}$ Edition, Marcel Dekker, Inc. 1994 